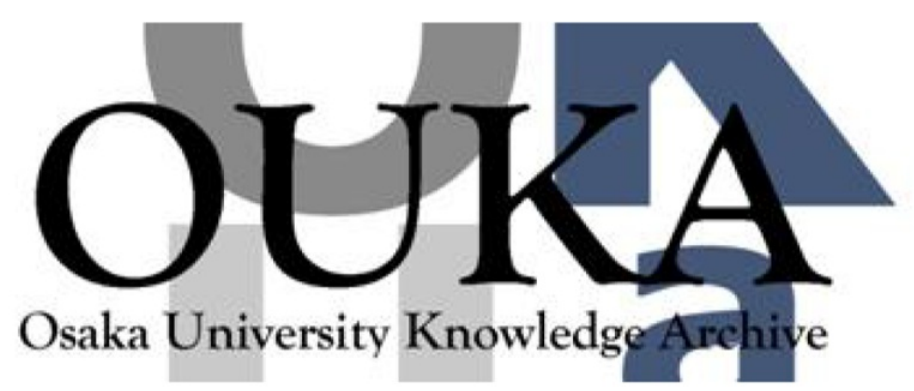

\begin{tabular}{|c|l|}
\hline Title & $\begin{array}{l}\text { Optical recording using smectic layer rotation } \\
\text { in ferroelectric liquid crystal }\end{array}$ \\
\hline Author(s) & $\begin{array}{l}\text { Nakayama, Keizo; Ohtsubo, Junji; Ozaki, } \\
\text { Masanori et al. }\end{array}$ \\
\hline Citation & Applied Physics Letters. 80(14) p. 2439-p. 2441 \\
\hline Issue Date & $2002-04-02$ \\
\hline oaire:version & VoR \\
\hline URL & https://hdl.handle.net/11094/75833 \\
\hline rights & \\
\hline Note & \\
\hline
\end{tabular}

Osaka University Knowledge Archive : OUKA

https://ir. Library. osaka-u. ac. jp/

Osaka University 


\section{Optical recording using smectic layer rotation in ferroelectric liquid crystal}

Cite as: Appl. Phys. Lett. 80, 2439 (2002); https://doi.org/10.1063/1.1467972

Submitted: 02 January 2002 . Accepted: 18 February 2002 . Published Online: 02 April 2002

Keizo Nakayama, Junji Ohtsubo, Masanori Ozaki, and Katsumi Yoshino

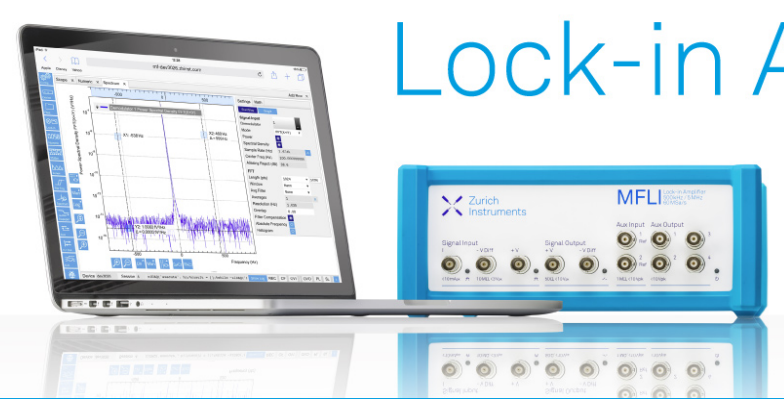

Amplifiers

Zurich

Instruments

Watch the Video 


\title{
Optical recording using smectic layer rotation in ferroelectric liquid crystal
}

\author{
Keizo Nakayama ${ }^{\text {a) }}$ and Junji Ohtsubo \\ Department of Systems Engineering, Faculty of Engineering, Shizuoka University, 3-5-1 Johoku, \\ Hamamatsu, Shizuoka 432-8561, Japan \\ Masanori Ozaki and Katsumi Yoshino \\ Department of Electronic Engineering, Graduate School of Engineering, Osaka University, \\ 2-1 Yamada-Oka, Suita, Osaka 565-0871, Japan
}

(Received 2 January 2002; accepted for publication 18 February 2002)

\begin{abstract}
Optical recording in a ferroelectric liquid crystal using smectic layer rotation induced by the application of asymmetric voltage pulses has been proposed. This recording method is based on the temperature dependence of the rotation rate and the fact that the rate in the smectic $\mathrm{A}(\mathrm{SmA})$ phase is considerably smaller than that in the chiral smectic $\mathrm{C}\left(\mathrm{SmC}^{*}\right)$ phase. The transition from the $\mathrm{SmC}^{*}$ to the SmA phase can be induced by the photothermal effect. The application of asymmetric voltage pulses during partial laser irradiation results in the patterning of the layer alignment. This recording method can erase and invert the stored pattern and can handle gray-level patterns.

(c) 2002 American Institute of Physics. [DOI: 10.1063/1.1467972]
\end{abstract}

Ferroelectric liquid crystals (FLCs) have a number of attractive properties for electro-optical applications, such as a fast electro-optical response, due to a direct interaction between the spontaneous polarization $\left(P_{S}\right)$ and an electric field, and the bistability of the switching of molecules in a thin cell. The bistability can be used to realize a memory function in an electro-optical device. Such devices are called surface-stabilized ferroelectric liquid crystals (SSFLCs) and have been extensively studied. In particular, the dynamics of the molecular switching in FLCs is a field of active research.

The dynamics of the smectic layer under the application of an electric field has also been researched. ${ }^{1-4}$ However, these reports mainly focus on the fundamental physical property or on the use of the reorientation to produce a FLC device with a uniform smectic layer alignment. On the other hand, we have researched controlling the smectic layer alignment and the use of smectic layer control as a mechanism adding functionality to a FLC device.

We have previously reported smectic layer rotation induced by the application of asymmetric voltage pulses in chiral smectic liquid crystals. ${ }^{5-7}$ Smectic layer rotation is of interest both as a physical phenomenon and for the purposes of practical applications, and other researchers have also researched the reorientation of the smectic layer. ${ }^{8-10}$ However, the layer rotation we have reported, generally seems to be a negative phenomenon for the application to electro-optical devices, in which a change in the layer alignment leads to unwanted changes in the optical intensity, specifically, a decrease in the contrast ratio or a change in the electro-optical response. The detail of this effect is shown in Fig. 1. The practical configuration of a SSFLC device is shown in Fig. 1(a). In Fig. 1(a), in the "down state" of the bistable states, the molecular long axis (director) is parallel to the polarizer and no light is transmitted; in the "up state" the light is transmitted. The director can be changed from one state to the other by reversing the sign of the applied electric field. A

a)Electronic mail: nakayama@sys.eng.shizuoka.ac.jp change in the director results in a switch between the dark and the bright states. If a smectic layer rotation occurs, e.g., with twice the molecular tilt angle, the electro-optical response of this device is completely inverted, as shown in Fig. 1(b). However, this layer rotation is continuous, reversible, and induced only by the application of an asymmetric voltage pulse. This suggests that the layer alignment is controllable through the application of asymmetric pulses. In a previous paper, we have proposed controlling the layer alignment using asymmetric pulses and have measured the change in the electro-optical response by layer rotation. ${ }^{11}$

Furthermore, we have proposed a method of optical recording, in which a pattern is stored as a partial alignment change of the smectic layer. ${ }^{12-14}$ This method uses a FLC

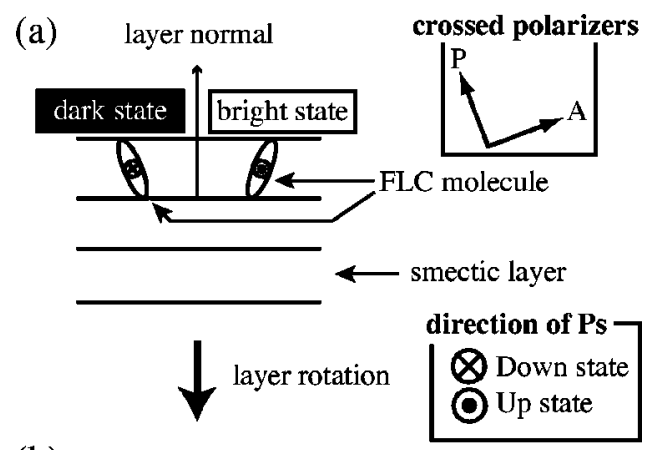

(b)

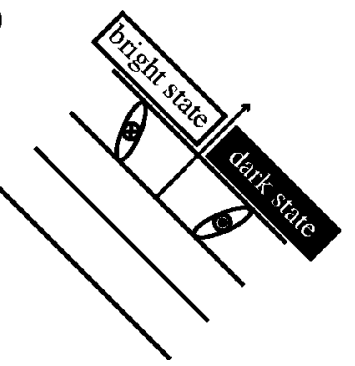

FIG. 1. Schematics of the configurations of the crossed polarizers, the smectic layer, and the molecular switching in case that the molecular tilt angle is $22.5^{\circ}$. (a) Practical configuration of the SSFLC device. (b) Configuration after a layer rotation of twice the molecular tilt angle. 


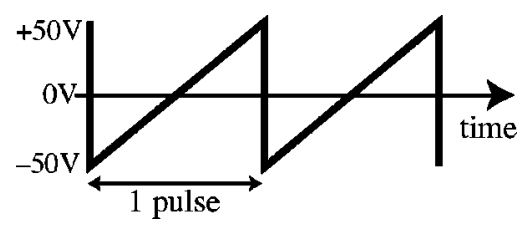

FIG. 2. Asymmetric voltage pulses used in this study.

having the chiral nematic $\left(\mathrm{N}^{*}\right)$-chiral smectic $\mathrm{C}\left(\mathrm{SmC}^{*}\right)$ phase sequence. Using this FLC, in the rubbing cell two kinds of domains with different layer alignments coexist in the $\mathrm{SmC}^{*}$ phase. When an external field is applied through a transition from the $\mathrm{N}^{*}$ to the $\mathrm{SmC}^{*}$ phase, one of the two domains can be obtained. In order to realize the optical recording method, it has been proposed to induce the phase transition by the photothermal effect ${ }^{12,13}$ and photoisomerization. ${ }^{14}$ Due to there being only two kinds of layer alignments, the optical-recording device using this FLC can store only a binary spatial pattern.

In the present letter, we propose an optical recording using smectic layer rotation. In order to realize the optical recording, the temperature dependence of the rotation rate is utilized. In this method we can use countless kinds of layer alignment controllable by the application of asymmetric voltage pulses.

The FLC used in this study is CS-1024 (Chisso) with the isotropic-N*-smectic A $(\mathrm{SmA})-\mathrm{SmC}^{*}$ phase sequence. The FLC was doped with G-239 (Hayashibara Biochemical Laboratories) dye of $1.3 \mathrm{wt} \%$ concentration so as to improve the efficiency of laser absorption. The cell used in this study was commercial (E. H. C.) and consisted of two indium-tinoxide-covered glass plates. The surfaces of the two glass plates were coated with a rubbed polyimide layer with the rubbing directions antiparallel. The cell gap was $10 \mu \mathrm{m}$. The temperature of the cell was controlled with a hot bath and a temperature controller. The applied voltage pulses were generated by an arbitrary waveform generator (Agilent, 33120A) and were amplified by a voltage amplifier (FLC Electronics, F20A). A diode-pumped crystal laser was used as a light source in the optical recording processes. The wavelength, output power, and transverse mode of the laser were $532 \mathrm{~nm}$, $100 \mathrm{~mW}$, and $\mathrm{TEM}_{00}$, respectively. Figure 2 shows the asymmetric voltage pulses used in this study. This sawtooth waveform has no dc component, and the asymmetry is in the polarity reversal; the polarity reversal from positive to negative is stepwise but slopes in the negative to positive polarity reversal.

The basic principle of the proposed method is to use laser irradiation to partially heat the sample through the photothermal effect. If the rotation rate depends on the temperature, the total rotation angles in the irradiated and nonirradiated areas are different after the application of asymmetric pulses during laser irradiation. The temperature dependence of the rotation rate was investigated first, using pure CS1024. The rotation rate was defined as the average angle of the layer rotation per asymmetric pulse after the application of 100 pulses. A sawtooth waveform, with a frequency of 3 $\mathrm{Hz}$, was used as an asymmetric voltage pulse, shown in Fig. 2. Figure 3 shows the rotation rate as a function of the reduced temperature relative to the transition temperature

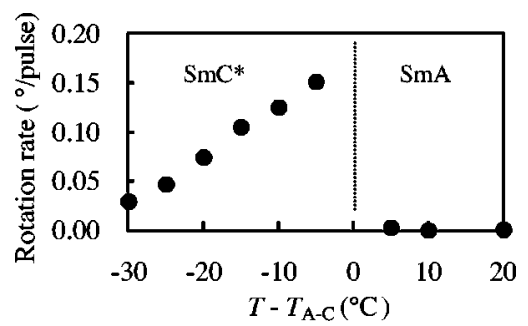

FIG. 3. Rotation rate as a function of temperature. Rotation rate is defined as the average rotation angle per asymmetric pulse.

$T_{A-C}$ from the $\mathrm{SmA}$ to the $\mathrm{SmC}^{*}$ phase. The rotation rate in the $\mathrm{SmA}$ phase is considerably smaller than in the $\mathrm{SmC}^{*}$ phase, and hence, the layer rotation in the SmA phase is negligible. Therefore, the change in the rotation rate was sharpest at $T_{A-C}$; in other words, the most efficient way to employ the temperature dependence is to utilize the change in the rotation rate at the phase transition.

The following method making use of the above observation is proposed. The temperature of the cell is kept just below $T_{A-C}$. When a part of the cell is irradiated with the laser beam, only the part of the sample in the irradiated area converts to the SmA phase through the photothermal effect [Fig. 4(a)]. Here, layer rotation is not induced by the application of asymmetric voltage pulses in the irradiated area, while layer rotation is induced in the nonirradiated area, in which the sample is in the $\mathrm{SmC}^{*}$ phase. Therefore, the application of asymmetric voltage pulses during the laser irradiation causes a change in the layer alignment only in the nonirradiated area, as shown in Fig. 4(b). After the laser irradiation is turned off, the whole of the sample is in the $\mathrm{SmC}^{*}$ phase. (Hereafter, this process is called the writing process.) The new layer alignment is normally stable under applying an electric field without asymmetry, namely, the layer alignment pattern achieved under irradiation is stored. Under the application of a dc field to this cell, the directions of the optical axes, which correspond to the directors of the molecule, are different in each area, since the molecules in each area tilt with respect to the layer normal. Accordingly, the difference in the layer alignments can be converted into a difference in optical intensity using crossed polarizers. In addition, the stored pattern can be erased, owing to the reversibility of layer rotation. In the erasing process the cell is
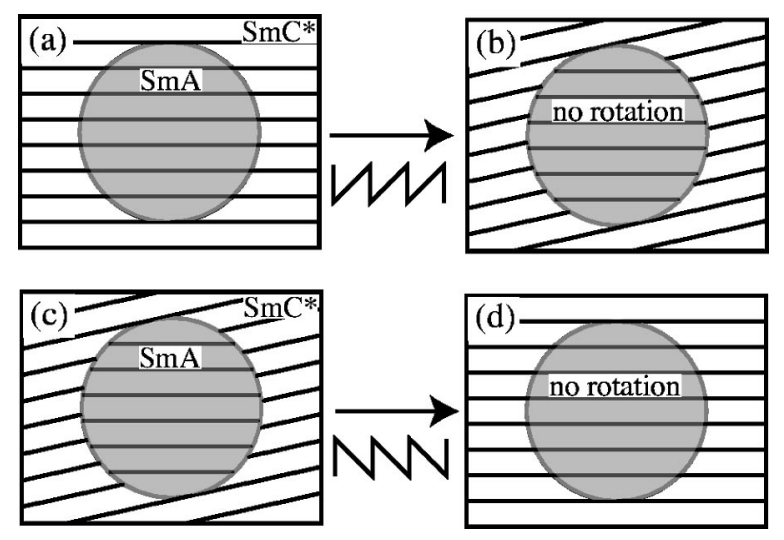

FIG. 4. Schematics of optical recording proposed in this study. Gray areas represent areas irradiated with the laser; (a) and (b) writing process and (c) and (d) erasing process. 


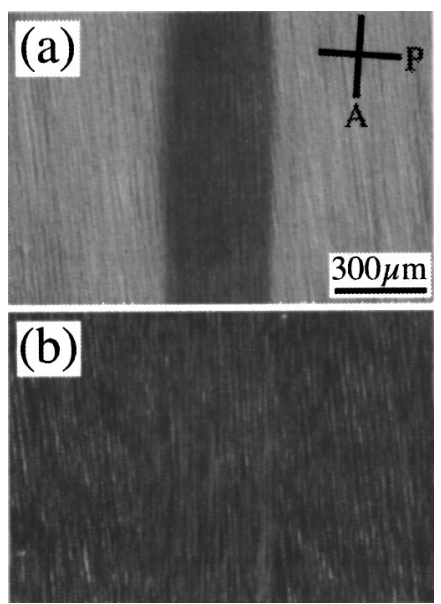

FIG. 5. Polarizing microphotographs under the application of a dc voltage of $20 \mathrm{~V}$; (a) after the writing process and (b) after the erasing process.

first irradiated with the laser. This changes the irradiated part of the SmA phase, as shown in Fig. 4(c). Then, asymmetric voltage pulses with the opposite polarity to those used in the writing process are applied during this laser irradiation. This causes the layer in the nonirradiated area to rotate in the opposite direction to the case of the writing process. As a result, the layer alignment returns to the initial state, as shown in Fig. 4(d).

We next tried out this method using CS-1024 doped with G-239. In this experiment, the cell was kept at $1{ }^{\circ} \mathrm{C}$ below $T_{A-C}$. The power of the incident laser beam into the cell was reduced to $65 \mathrm{~mW}$ with a neutral density filter. The profile of the laser beam on the sample through a mask was a rectangle (note that the profile of the original laser beam without using the mask was a circle with a diameter of $2.3 \mathrm{~mm}$ ). The frequency of the sawtooth pulse used, as shown in Fig. 2, was 5 $\mathrm{Hz}$. The number of applied pulses used was 50 in the writing process and 25 in the erasing process. The difference is necessary because the rotation rate depends on the rotation direction in a rubbing cell. ${ }^{11}$ Figure 5(a) shows a polarizing microphotograph after the writing process. The dark region is the irradiated area, that is, the layer alignment in this area is not changed by the application of asymmetric voltage pulses. In the bright area, on the other hand, a counterclockwise layer rotation of $5.9^{\circ}$ is induced. The erasing process was carried out subsequently and the result is shown in Fig. 5(b). The whole area looks largely uniform in optical intensity, showing that the stored pattern was erased.

This method has a number of merits. Since the layer rotation is continuous, gray-level patterns can be handled in this method. Furthermore, in devices using FLCs with a tilt angle of $22.5^{\circ}$ we are able to switch the visualized pattern between a negative and a positive image of the stored pattern by simply inverting the polarity of the applied dc field in reading the stored pattern. The use of smectic layer rotation for optical recording can be realized by other optical recording methods: for example, a method where the phase transition induced by photoisomerization or a method where a photoconductor is used as an electrode of the cell.

We have proposed and demonstrated an optical recording method in a FLC using smectic layer rotation induced by the application of asymmetric voltage pulses. This optical recording method can erase and invert the stored pattern and can handle gray-level patterns.

One of the authors (K.N.) was supported by a Grant-inAid for Encouragement of Young Scientists (No. 13750043) from the Japan Society for the Promotion of Science.

${ }^{1}$ J. S. Patel, S.-D. Lee, and J. W. Goodby, Phys. Rev. A 40, 2854 (1989).

${ }^{2}$ J. S. Patel and J. W. Goodby, J. Appl. Phys. 59, 2355 (1986).

${ }^{3}$ T. Hatano, K. Yamamoto, H. Takezoe, and A. Fukuda, Jpn. J. Appl. Phys., Part 1 25, 1762 (1986).

${ }^{4}$ K. Myojin, H. Moritake, M. Ozaki, K. Yoshino, T. Tani, and K. Fujisawa, Jpn. J. Appl. Phys., Part 1 33, 5491 (1994).

${ }^{5}$ M. Ozaki, H. Moritake, K. Nakayama, and K. Yoshino, Jpn. J. Appl. Phys., Part 2 33, L1620 (1994).

${ }^{6}$ K. Nakayama, H. Moritake, M. Ozaki, and K. Yoshino, Jpn. J. Appl. Phys., Part 2 34, L1599 (1995).

${ }^{7}$ K. Nakayama, M. Ozaki, and K. Yoshino, Jpn. J. Appl. Phys., Part 1 35, 6200 (1996)

${ }^{8}$ I. Dierking, L. Komitov, and S. T. Lagerwall, Jpn. J. Appl. Phys., Part 2 37, L57 (1998).

${ }^{9}$ I. Dierking, L. Komitov, and S. T. Lagerwall, Jpn. J. Appl. Phys., Part 2 37, L525 (1998).

${ }^{10}$ I. Dierking, B. Glusen, S. T. Lagerwall, and C. K. Ober, Phys. Rev. E 61, 1593 (2000).

${ }^{11}$ K. Nakayama, M. Ozaki, and K. Yoshino, Jpn. J. Appl. Phys., Part 1 36, 6122 (1997).

${ }^{12}$ K. Nakayama, M. Ozaki, and K. Yoshino, Jpn. J. Appl. Phys., Part 1 37, 5379 (1998).

${ }^{13}$ K. Nakayama, M. Ozaki, and K. Yoshino, Mol. Cryst. Liq. Cryst. 331, 273 (1999).

${ }^{14}$ T. Matsui, K. Nakayama, M. Ozaki, and K. Yoshino, Appl. Phys. Lett. 76, $1228(2000)$ 